\title{
Imaging and Dosimetric Consideration for Titanium Prosthesis Implanted within the Irradiated Region by Cobalt-60 Teletherapy Unit
}

\author{
Vaino Indongo ${ }^{1 *}$, Samuel Nii Adu Tagoe ${ }^{2}$, Kwame Kyere ${ }^{3}$, Cyril Schandorf ${ }^{3}$ \\ ${ }^{1}$ Namibia University of Science and Technology (NUST), Windhoek, Namibia \\ ${ }^{2}$ Radiotherapy and Nuclear Medicine Centre, Korle Bu Teaching Hospital, Accra, Ghana \\ ${ }^{3}$ School of Health and Allied Sciences, University of Ghana, Accra, Ghana \\ Email: *vindongo@nust.na
}

How to cite this paper: Indongo, V., Tagoe, S.N.A., Kyere, K. and Schandorf, C. (2018) Imaging and Dosimetric Consideration for Titanium Prosthesis Implanted within the Irradiated Region by Cobalt-60 Teletherapy Unit. International Journal of Medical Physics, Clinical Engineering and Radiation Oncology, 7, 160-172.

https://doi.org/10.4236/ijmpcero.2018.72014

Received: February 27, 2018

Accepted: May 5, 2018

Published: May 8, 2018

Copyright $\odot 2018$ by authors and Scientific Research Publishing Inc. This work is licensed under the Creative Commons Attribution International License (CC BY 4.0).

http://creativecommons.org/licenses/by/4.0/

\section{(c) (i) Open Access}

\begin{abstract}
The aim of this research is to observe dose distributions in the vicinity of titanium prosthetic implants during radiotherapy procedures on ${ }^{60} \mathrm{Co}$ teletherapy machine, Prowess Panther treatment planning system (TPS). Data were obtained using a locally fabricated tissue equivalent phantom CT images with titanium prosthesis which was irradiated with ${ }^{60} \mathrm{Co}$ gamma radiation. Prowess TPS $(1.25 \mathrm{MeV})$ estimated less variations. Proximal ends of the metal recorded slight increase in doses as a result of backscatter with dose increment below acceptable tolerance of $\pm 3 \%$. Doses measured decreases on the distal side of the prosthesis at a distance less than $\mathrm{d}_{\max }$ from the plate on each beam energy. The depth dose increases marginally after a certain depth level which generally originated from the unperturbed dose due to increase in the electron fluence. The percentage of depth doses decrease with the increase in plate thickness. A reduction in the above trend was also noticed with an increase in beam energy primarily because scattered photons are more forwardly directed. Prowess TPS (convolution superposition algorithm) was found to be better at reducing dose variation when correction for artifact. Manual calculations on blue phantom data agree with results from Prowess. This treatment system is capable of simulating dose around titanium prosthesis as its range of densities, 0.00121 to 2.83 , excludes titanium density (rED for titanium is 3.74).
\end{abstract}

\section{Keywords}

Phantom, Co-60, Dosimetric, Imaging, Titanium, Implant, Prosthesis, Prowess Panther 


\section{Introduction}

Radiotherapy is a treatment of cancer with ionizing radiations. Radiotherapy is classified into two main areas, namely: external beam radiation therapy (teletherapy); where the source of radiation is in remote from the patient's region to be treated and brachytherapy; where radiation sources are placed directly or in close proximity to the target to be treated in the host. With reference to this, image-based radiation therapy has become the standard practice. Patient data are acquired with computed tomography (CT) scanners, and then exported or downloaded into the Treatment Planning System (TPS) for dose computation to obtain dose distribution within the treated region. Optimization of the radiation dose to the intended target is achieved with the TPS by selecting appropriate beam irradiation geometries and beam weightings [1] [2].

This work focuses on titanium prostheses and their effects on CT imaging and radiation metrology. The best practice is to consider the dosimetric effects of this prosthetic implant in order to enhance the accuracy in the patient radiation dose delivered such that it meets the tolerance of $\pm 3 \%$ (difference between measured and calculated dose) as recommended by International Commission on Radiation Units \& Measurements (ICRU) for regions implanted with prostheses [3] $[4]$.

\section{Methods and Materials}

\subsection{Materials}

The materials used in this study include the following: Equinox 100 Cobalt-60 teletherapy machine (Best Theratronics), locally designed (fabricated) water phantom to represent the pelvic region or trunk of an average adult human, ionization chambers, electrometers, barometer, digital thermometer, Titanium for commercial use (Grade 1; C: 0.01, Fe: 0.097, N: 0.014, $\mathrm{O}_{2}: 0.099, \mathrm{H}: 0.002$, Total < 0.4) plates, CT scanners, tissue characterization phantom, Blue water phantom, Treatment Planning System (Prowess Panther Version 4.6) and RadiAnt DICOM Viewer software.

\subsection{Water Phantoms}

Plate 1 shows a pictorial view of the round phantom used for this study. The phantom was marked on the laterals and anterior with cross marks where lasers pass through during measurements on both CT scanner and teletherapy machines. Titanium plates of dimensions of $400 \times 40 \times 4 \mathrm{~mm}^{3}$ (length $\times$ breadth $\times$ width) were submerged in water for measurements.

Another full scatter blue water phantom of dimensions $480 \times 480 \times 410 \mathrm{~mm}^{3}$ was also used (Plate 2). This is a measuring device for the measurements and analysis of the radiation beam of medical linear accelerator which use Omni-Pro Accept software for analyzing the measured data. It consists of a $3 \mathrm{D}$ servo (the blue water phantom with mechanics), a common control unit (CCU) with integrated two-channel electrometer and two single detectors (ionization chambers). 


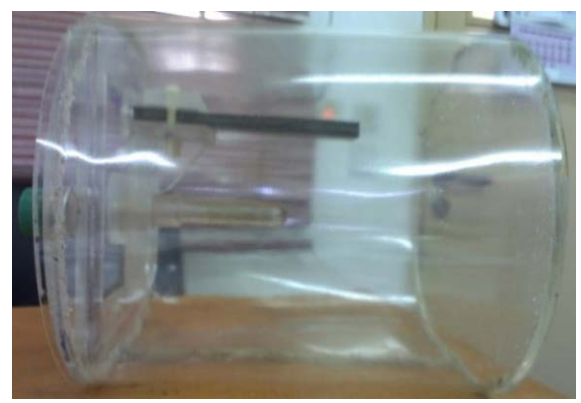

Plate 1. A pictorial view of the PMMA in-house built water phantom.

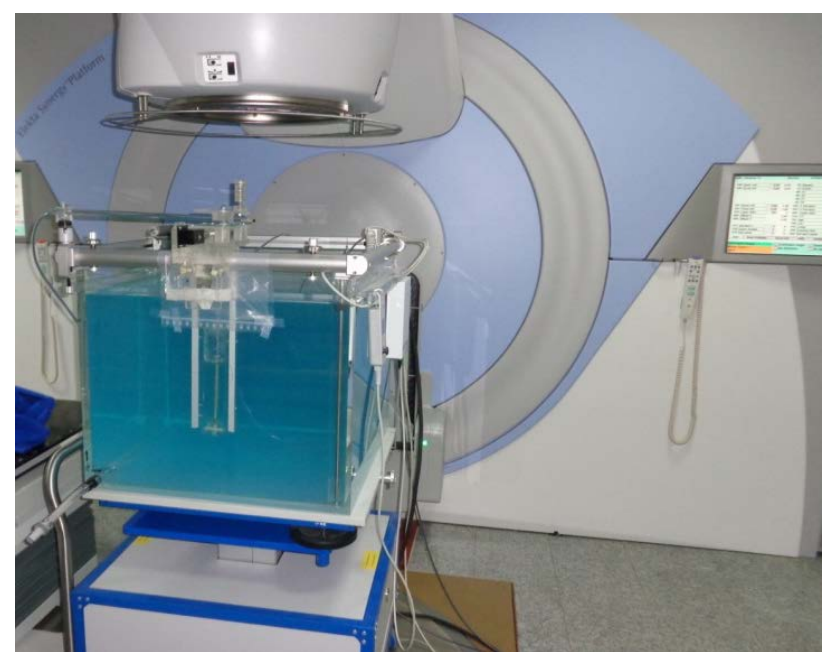

Plate 2. The blue water phantom setups under Elekta Synergy Platform gantry.

Basic dose distribution data are usually measured in a water phantom, and this closely approximates the radiation absorption and scattering properties of muscle and other soft tissues. In addition to that, the choice of water as a phantom material is that it is universally available with reproducible radiation properties. A well-constructed titanium holder was then mounted on the edge of the blue water phantom.

\subsection{Scanning of Phantom}

The tissue characterization phantom was scanned with both CT scanners. Prior to the scanning of the phantom it was insured that the phantom was positioned such that it was central to the aperture of the CT scanners. This was done with the help of internal patient alignment system (lasers) of the CT scanners and three indentation marks placed on the phantom. After scanning the phantom in each case, a titanium insert (Plate 3) was used to replace one of the inserts and the scan repeated. The positioning of the titanium insert was replaced with another insert such that the titanium insert was moved to the inner concentric ring and the scan was repeated. The change of position of the titanium insert within the phantom was to account the effect of the position of a material on its CT number [1] [5]. The bigger phantom was dismantled and the head phantom was removed. The above scanning procedure was repeated with head phantom. 
For the head phantom the position of the titanium on the outer concentric ring was replaced by another insert in the center of the phantom. Some scan parameters were used during the scanning of the tissue characterization phantom; 120 $\mathrm{kVp}$ for scans with the 6 slice CT scanner. For the scans the slice thickness was set at $2.5 \mathrm{~mm}$ for a CT scanner. After the scans, the CT image data sets of the phantoms were downloaded on to a CD-ROM.

\subsection{Determination of CT Numbers}

The CT image data sets on the CD-ROM were read with RadiAnt DICOM Viewer to determine the HU of the various inserts within the phantoms. A circular region of interest (ROI) with an area equal to one fourth the total cross-sectional area of an insert was created. For the titanium insert the ROI was smaller, this was because the titanium within the insert was very small as shown in Plate 4. The aim of a small size was to avoid many streak artifacts in the

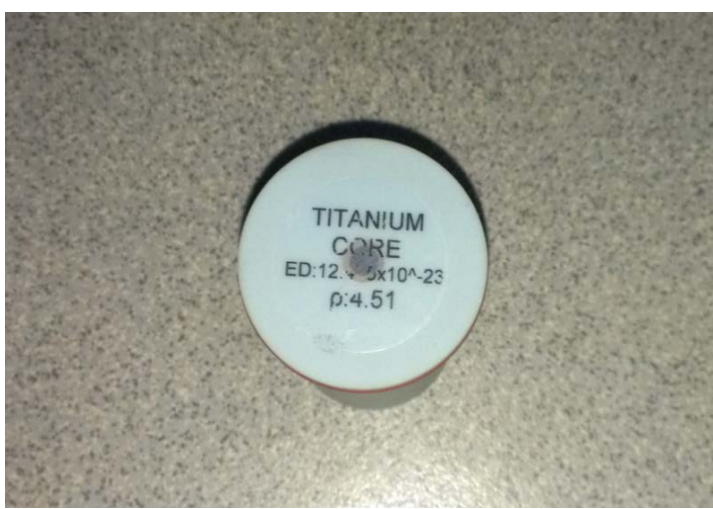

Plate 3. The Titanium insert, with a density $(\rho)$ of $4.51 \mathrm{~g} / \mathrm{cm}^{3}$ and electron density (ED) of $12.475 \times 10^{23}$ per $\mathrm{cm}^{3}$.

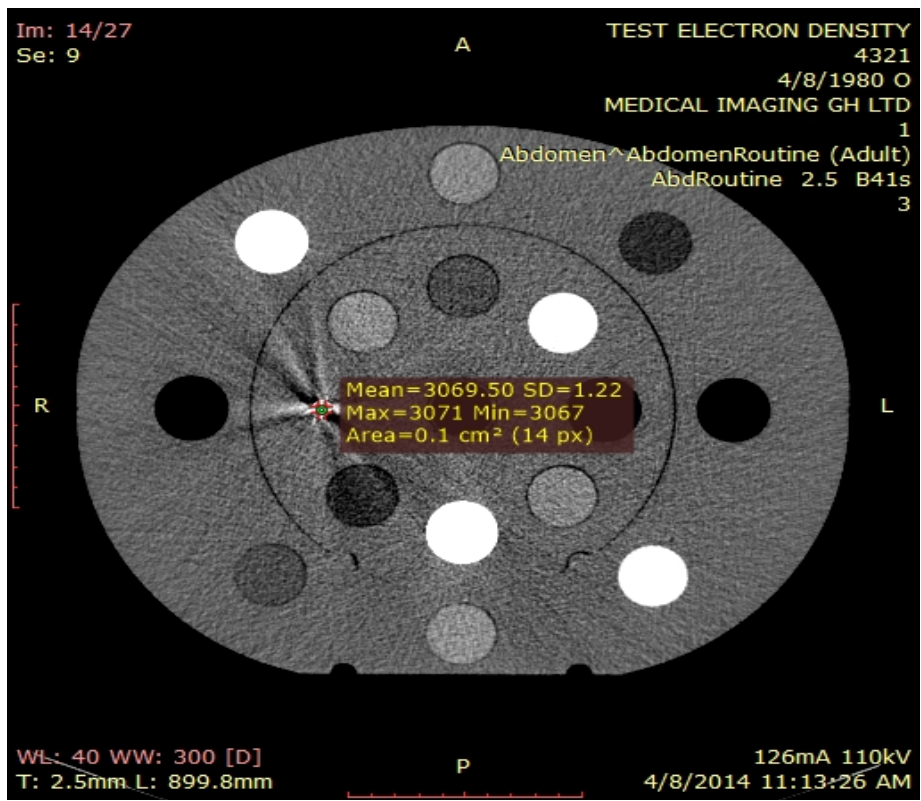

Plate 4. An approximated HU of titanium inset on a selected slice, abdomen scan routine. 
image, which also causes high discrepancies on HU of other inserts. This ROI was used to measure $\mathrm{HU}$ of the insert such that the ROI was central to the insert during the measurement. These procedures were repeated for a number of successive slices per phantom. The mean $\mathrm{HU}$ values $\left(\mathrm{HU}_{\mathrm{m}}\right)$ of the region encompass by $\mathrm{ROI}$ was displayed and recorded. The average $\mathrm{HU}_{\mathrm{m}}$ for the same insert was determined regardless of its position and phantom type. The average $\mathrm{HU}_{\mathrm{m}}$ values determined were correlated against their respective electron densities inscribed on the surface of an insert. From this correlation CT number to relative electron density convention curves were shown for each individual CT scanner [5].

A picture (Plate 4) showing the approximate Hounsfield Units above was obtained from the RadiAnt DICOM Viewer. For this particular slice, potential energy was $110 \mathrm{kVp}$ and tube current of $126 \mathrm{~mA}$. The mean CT number obtained was identified as 3069 with standard deviation of 1.22 and the region of interest was 14 pixels.

\subsection{An In-House (Fabricated) Phantom Imaging}

CT scanner was used to scan the locally fabricated water phantom which had a holder within it to hold different configurations of titanium plates in place. The holder was designed such that the position of the titanium plate from the surface of the phantom can be altered as shown in Plate 5. Before the CT scans were performed, the phantom was set up such that the laser passed through the cross markings on the phantom. Typical scanning parameter of $120 \mathrm{kVp}$ and $130 \mathrm{kVp}$ were used in standard scanning protocols for the 6 slice scanner. The slice thicknesses were set at $3 \mathrm{~mm}$ for both scanners. After the scan, CT image data with $\mathrm{CT}$ scanner were copied into the compact disk (CD) and imported into the

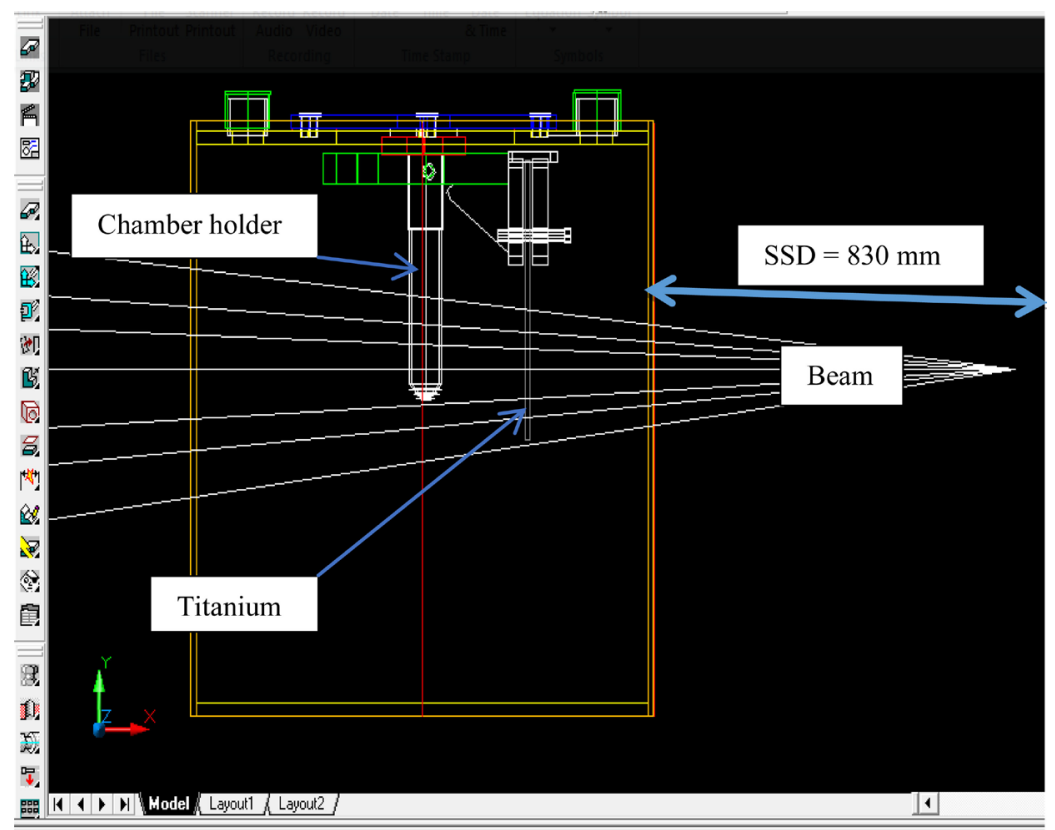

Plate 5. Experimental setup and simulation geometry for acquisition of depth dose data in water, with a titanium plate $\left(\rho=4.65 \mathrm{~g} / \mathrm{cm}^{3}\right), 4 \mathrm{~mm}$ thickness [6]. 
Prowess Panther 4.6 treatment planning systems.

\subsection{Dose Measurements in Round Phantom}

Forward planning was done in both TPS with a prescribed dose of 2 Gy (200 cGy) normalized to center of the sensitive volume of the chamber inserted within the phantom. SAD treatment technique was employed using various field sizes $\left(7 \times 7 \mathrm{~cm}^{2}, 10 \times 10 \mathrm{~cm}^{2}\right.$, and $\left.15 \times 15 \mathrm{~cm}^{2}\right)$ and the treatment time was recorded for all irradiation geometries. The beam came from the left lateral side such that the direction of propagation of the beam was perpendicular to the titanium plate within the phantom (Plate 6).

\section{Results and Discussions}

\subsection{Computed Tomography Images}

Axial images of the tissue characterization phantom scanned with SOMATOM Emotion CT scanner, during the CT calibration, are shown in Plate 7. Plate 8 shows transverse images of the phantom scanned with and without titanium insert in the phantom. The image with titanium insert is affected by streak artifacts due to high variation in density between the metal and the other materials in close proximity to the metal. The metal attenuates most of the X-rays through photoelectric effect as a result of the energy of the beam used for the CT scan. The intensity of the X-rays after passing through the metal is very low, which will make transmitted X-rays from neighbouring materials low than they are truly supposed. Materials in the neighbourhood of the metal may have densities or HU far low than normal. With reference to the above, it is therefore imperative to obtain the HUs of the various inserts without the titanium insert. The scan with the titanium insert was only used to determine $\mathrm{HU}$ of the titanium. The CT numbers were recorded in Table 1 for comparisons. The effect (streak artifacts) was found to improve with certain scan parameters such as increase in $\mathrm{kVp}$ and beam filtration applied in the CT scanner. These were in consonance with studies done by other researchers [5].

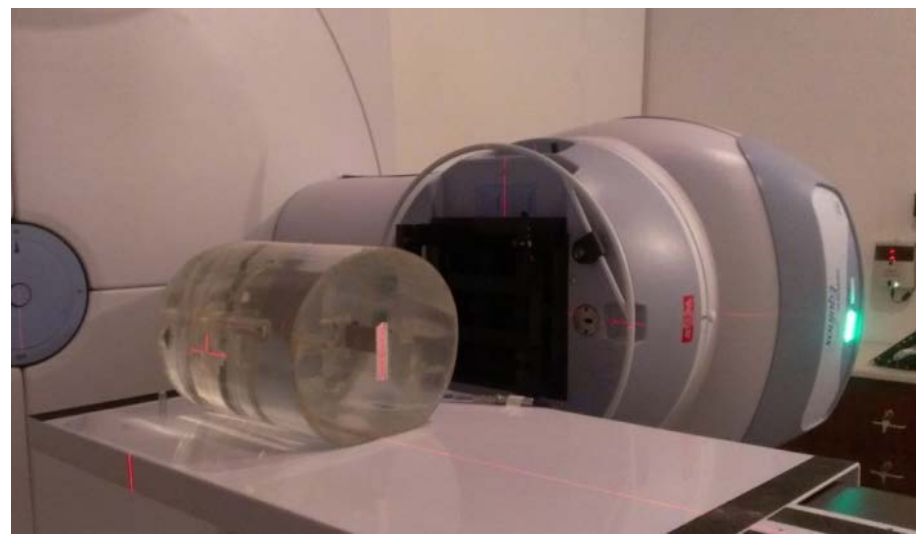

Plate 6. The round phantom orientation on the couch during measurement of TPS planned doses, with vertical and horizontal lasers and ${ }^{60} \mathrm{Co}$ gantry at $90^{\circ}$. 


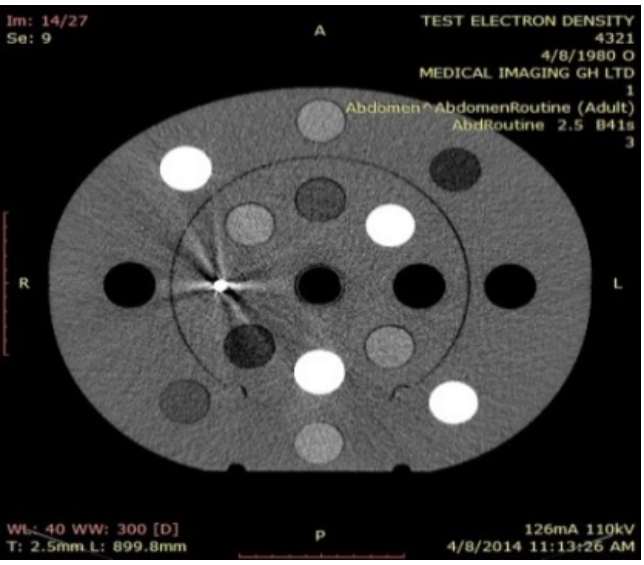

(a)

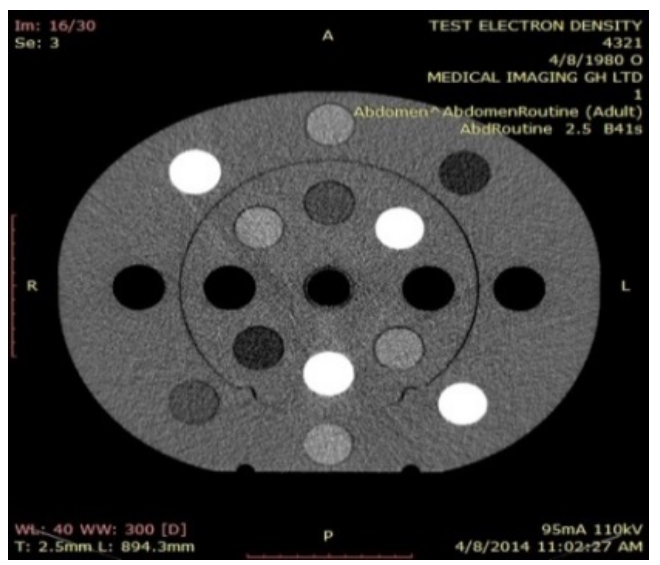

(b)

Plate 7. Axial view of a tissue characterization phantom, (a) without and (b) with titanium insert surrounded by streak artifacts.

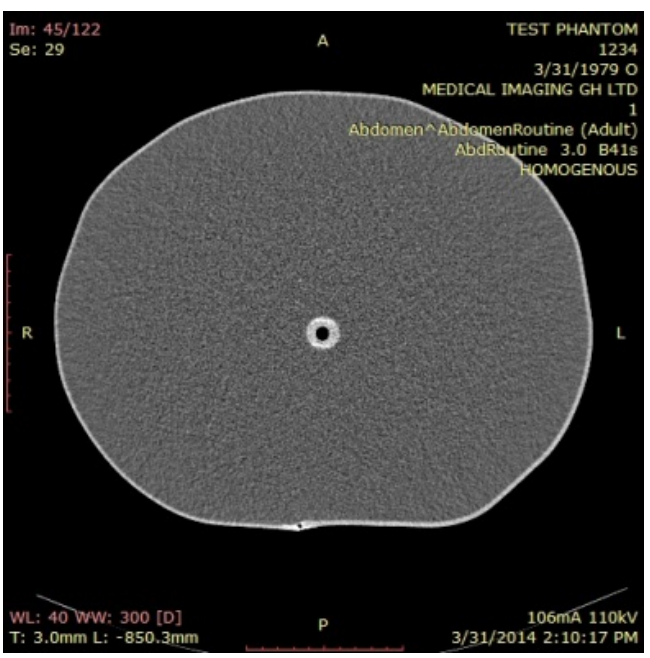

(a)

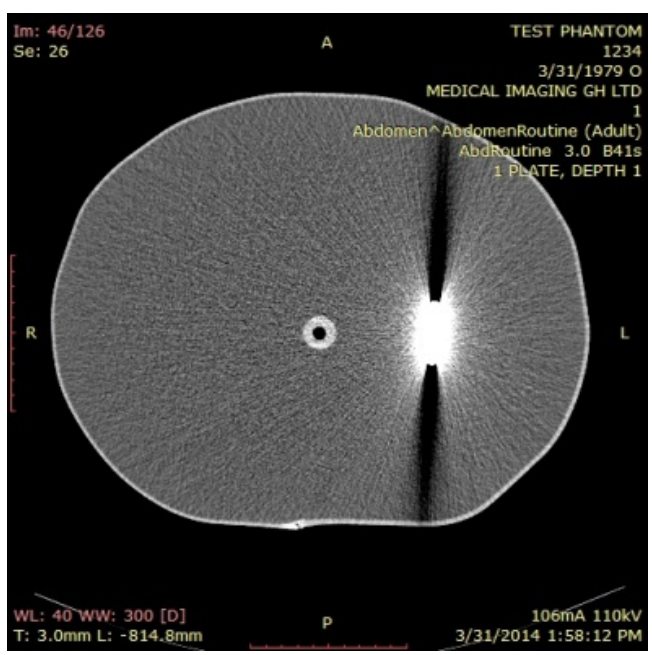

(b)

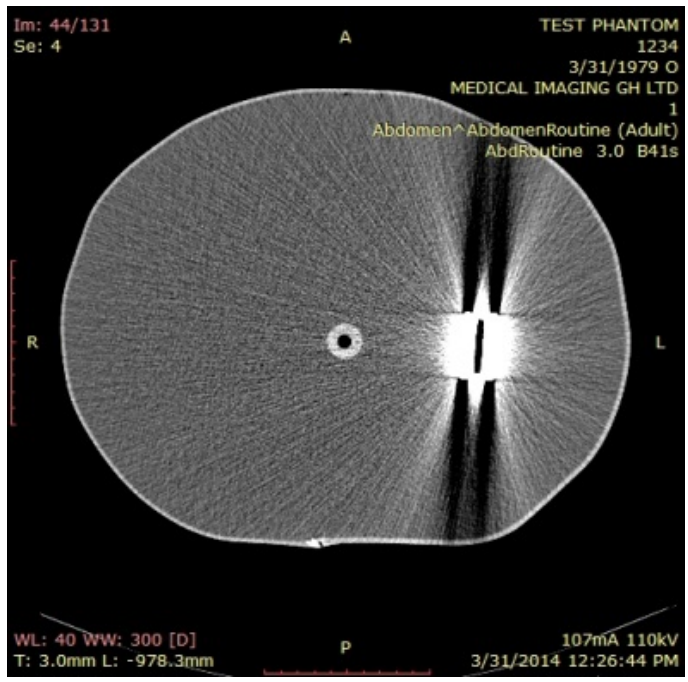

(c)

Plate 8. Axial images of a fabricated water phantom showing (a) homogeneous (left), (b) 4 mm titanium (centre) and (c) hollow (air gap) metal (right) scans. 
Table 1. Comparison between CT numbers measured with tissue characterization phantom and those of CT number to rED conversion curves for Prowess Panther TPS for the requisite CT scanner.

\begin{tabular}{|c|c|c|c|c|c|}
\hline \multirow{2}{*}{$\begin{array}{l}\text { Part Number } \\
\text { of Insert }\end{array}$} & \multirow{2}{*}{$\begin{array}{l}\text { Description of Insert } \\
\text { (Simulating Tissue) }\end{array}$} & $\begin{array}{l}\text { Electron } \\
\text { Densities }\end{array}$ & CT Values & $\begin{array}{c}\text { Measured } \\
\text { HUs }\end{array}$ & Abs. Diff. in $\mathrm{HU}$ \\
\hline & & $\mathrm{rED}$ & Prowess & 6-Slice & M6-Slice-Prow. \\
\hline- & Air & 0.001 & -997.80 & -987.26 & 10.55 \\
\hline 062A-04 & Lung (Inhale) & 0.19 & -826.32 & -831.97 & 5.65 \\
\hline 062A-05 & Lung (Exhale) & 0.49 & -492.82 & -493.59 & 0.77 \\
\hline $062 \mathrm{~A}-11$ & Adipose & 0.95 & -50.39 & -68.27 & 17.88 \\
\hline 062A-06 & Breast & 0.98 & -39.35 & -26.66 & 12.69 \\
\hline 062A-39 & Water & 1 & -11.57 & 1.85 & 13.42 \\
\hline 062A-10 & Muscle & 1.04 & 46.52 & 38.24 & 8.28 \\
\hline 062A-09 & Liver & 1.05 & 54.06 & 53.32 & 0.74 \\
\hline 062A-08 & Bone $(200 \mathrm{mg} / \mathrm{cc})$ & 1.12 & 336.92 & 271.78 & 65.14 \\
\hline 062A-15 & Dense Bone $(800 \mathrm{mg} / \mathrm{cc})$ & 1.46 & 995.83 & 982.99 & 12.84 \\
\hline $062 \mathrm{~A}-27$ & Bone $(1250 \mathrm{mg} / \mathrm{cc})$ & 1.7 & 1339.58 & 1358.97 & 19.39 \\
\hline $062 \mathrm{~A}-19^{* *}$ & ICRU Cortical Bone & 1.78 & 1516.67 & - & - \\
\hline 062A-29 & $\begin{array}{l}\text { Solid Dense Bone } \\
(1750 \mathrm{mg} / \mathrm{cc} \mathrm{HA})\end{array}$ & 1.95 & 1824.00 & - & - \\
\hline- & - & 2.15 & 2224.00 & - & - \\
\hline- & - & 2.34 & 2640.00 & - & - \\
\hline- & - & 2.46 & 2832.00 & - & - \\
\hline $062 \mathrm{~A}-12^{* *}$ & Titanium (Ti) & 3.74 & - & 2500 & 3068.41 \\
\hline
\end{tabular}

\subsection{Simulation of Effect of Prosthesis on Dose Distribution in Phantom Designed to Represent the Trunk or Pelvic Region with Cobalt-60 Beam}

Relative dose measurements at depth of $17 \mathrm{~cm}$ in the fabricated phantom without the prosthesis are within $\pm 3 \%$ of those calculated with the Prowess TPS for the selected field sizes for cobalt-60 beam. An air medium (hollow) created between the metal plates gave extra doses at the point of measurement. Calculated treatment time in the presence of hollow or air gap is more than the expected time needed for treatment, which may cause overdose to patients with prostheses. The measured doses compare to those calculated with the TPS increase as much as $6.5 \%$ for deep seated prosthesis. These results are shown in Figure 1. The effect is more pronounced with small field sizes. For smaller field sizes much of the radiation field is covered by the prosthesis and much of the radiation is attenuated. The TPS therefore exaggerates the attenuation offered by the prosthesis due to inherent deficiencies in the dose computation engine of the TPS or artifacts offsetting densities of the various materials constituting the phantom. This may also be as a result of enhancement of beam intensity and 


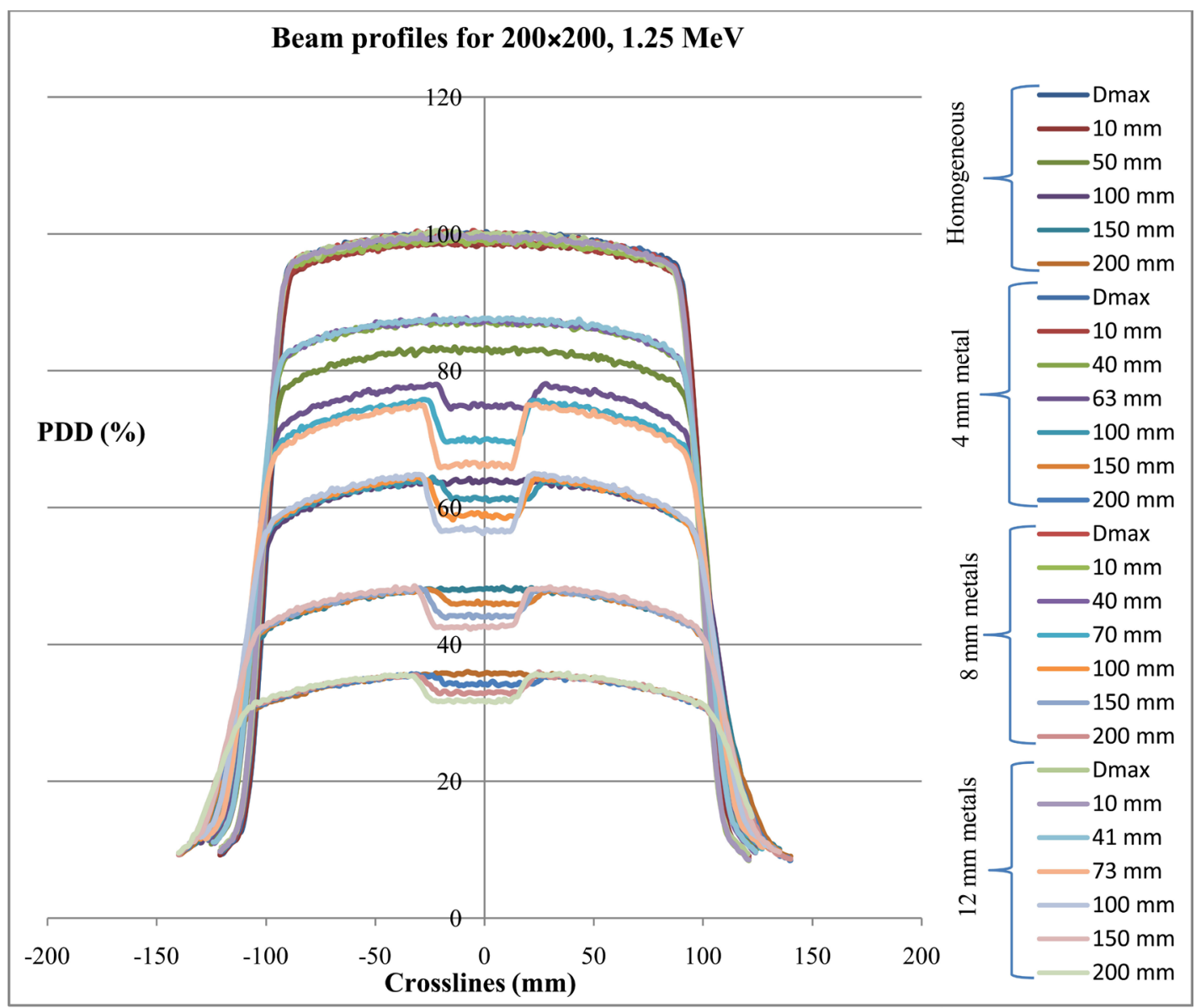

Figure 1. Measured PDDs of FS $=200 \times 200 \mathrm{~mm}^{2}$ superimposed for 4, 8 and $12 \mathrm{~mm}$ metal thicknesses irradiated with ${ }^{60} \mathrm{Co}$ beam energy.

incorrectly interpolated CT values around the region of hollowness. Reft et al. (2003) mentioned that the vital physical properties that can influence dosimetry of a radiation beam are; hollowness, shape, size, and composition of the material [3]. Hollow metals have been found to create a lot of artifacts in their vicinity on CT images and this may introduce errors in the doses calculated by the treatment planning system when a radiation beam is allowed to pass through this region.

The results show that for smaller field size, $7 \times 7 \mathrm{~cm}^{2}$, the doses measured by a detector at a given depth were enhanced less than doses at bigger field sizes. Thinner titanium plate $(4 \mathrm{~mm})$ attenuates less gamma rays than thicker plates. Shallow depth metal had less influence on the dose due to the fact that the streak artifacts are shallow and close to the surface, covering less medium area. The dose increase was between $1.1 \%$ and $4.5 \%$. In the presence of artifacts, attenuation is inconsistent and the doses measured at a point have a tremendous increase. Therefore, there is a likely overdose to OAR's located behind, in the region around the prosthetic material and in the beam perspective. Furthermore, the streak artifacts may obscure tumours as discussed by Shimozato et al. (2010) and OAR on the laterals of the metal, causing inappropriate control of cancer 
tumours during radiation therapy. The fact is that, artifacts caused by the presence of a metal in a volume scanned by an X-ray CT scanner may influence the CT numbers and eventually introduce errors in the dose distribution calculated [4] [7] [8]. It is a result of different CT numbers, more side scatter and forward scatter due to interactions of photons with a high dense material. Hence, convolution superposition calculation algorithm shows larger variations in simulation of dose distribution around the metal prosthesis in the presence of artifacts. Details on percentage deviations on measured dose to the calculated dose are shown in Table 2. Depth 1 is a depth of the detector from the distal surface of the metal when the metal holder fixed inside the phantom during measurements is at hole number 1 and it is approximately $7.0 \mathrm{~cm}$. Depth 2 is a depth of the chamber from distal side of the plate when the holder is fixed at hole number 2 and it is approximately $11.3 \mathrm{~cm}$. Plate 9 shows the distances when the metal holder is at depth 1 and 2.

After corrections for artifacts [9], the dose distribution varied between $-0.59 \%$ for a $7 \times 7 \mathrm{~cm}^{2}$ and $0.31 \%$ for the $15 \times 15 \mathrm{~cm}^{2}$ (within $\pm 3 \%$ tolerance) within a homogeneous medium. Table 3 shows all calculated values obtained after artifacts are corrected in Prowess Panther TPS. For the air gap created between the metal plates, there is still a large increase in dose at a point. The change in dose

Table 2. Measured dose for a phantom BEFORE correction of artifacts on ${ }^{60} \mathrm{Co}$ machine, and a calculated dose of $200 \mathrm{cGy}$.

\begin{tabular}{|c|c|c|c|c|c|c|}
\hline \multicolumn{7}{|c|}{ BEFORE the corrections for artifacts $\left({ }^{60} \mathrm{Co}\right.$ beam $)$} \\
\hline \multicolumn{7}{|l|}{ Depth 1} \\
\hline \multirow{2}{*}{$\begin{array}{l}\text { Thickness } \\
(\mathrm{mm})\end{array}$} & \multicolumn{3}{|c|}{ Measured dose for a field size (cGy) } & \multicolumn{3}{|c|}{$\begin{array}{c}\% \text { deviation between measured } \\
\text { and calculated }\end{array}$} \\
\hline & $7 \times 7 \mathrm{~cm}^{2}$ & $10 \times 10 \mathrm{~cm}^{2}$ & $15 \times 15 \mathrm{~cm}^{2}$ & $7 \times 7 \mathrm{~cm}^{2}$ & $10 \times 10 \mathrm{~cm}^{2}$ & $15 \times 15 \mathrm{~cm}^{2}$ \\
\hline 0 & 199.630 & 200.000 & 200.530 & -0.18 & 0.00 & 0.26 \\
\hline 4 & 207.270 & 207.460 & 207.060 & 3.64 & 3.73 & 3.53 \\
\hline 8 & 207.330 & 209.040 & 208.130 & 3.66 & 4.52 & 4.06 \\
\hline 12 & 204.910 & 206.280 & 205.890 & 2.45 & 3.14 & 2.94 \\
\hline 8 (air gap) & 212.980 & 212.700 & 209.920 & 6.49 & 6.35 & 4.96 \\
\hline \multicolumn{7}{|l|}{ Depth 2} \\
\hline \multirow{2}{*}{$\begin{array}{l}\text { Thickness } \\
\text { (mm) }\end{array}$} & \multicolumn{3}{|c|}{ Measured dose for a field size (cGy) } & \multicolumn{3}{|c|}{$\begin{array}{c}\% \text { deviation between measured } \\
\text { and calculated }\end{array}$} \\
\hline & $7 \times \quad 7 \mathrm{~cm}^{2}$ & $10 \times 10 \mathrm{~cm}^{2}$ & $15 \times 15 \mathrm{~cm}^{2}$ & $7 \times 7 \mathrm{~cm}^{2}$ & $10 \times 10 \mathrm{~cm}^{2}$ & $15 \times 15 \mathrm{~cm}^{2}$ \\
\hline 0 & 199.630 & 200.000 & 200.530 & -0.18 & 0.00 & 0.26 \\
\hline 4 & 204.090 & 204.800 & 204.790 & 2.04 & 2.40 & 2.40 \\
\hline 8 & 205.250 & 206.680 & 206.700 & 2.62 & 3.34 & 3.35 \\
\hline 12 & 199.020 & 201.420 & 201.470 & -0.49 & 0.71 & 0.74 \\
\hline 8 (air gap) & 205.350 & 206.270 & 206.450 & 2.68 & 3.13 & 3.23 \\
\hline
\end{tabular}




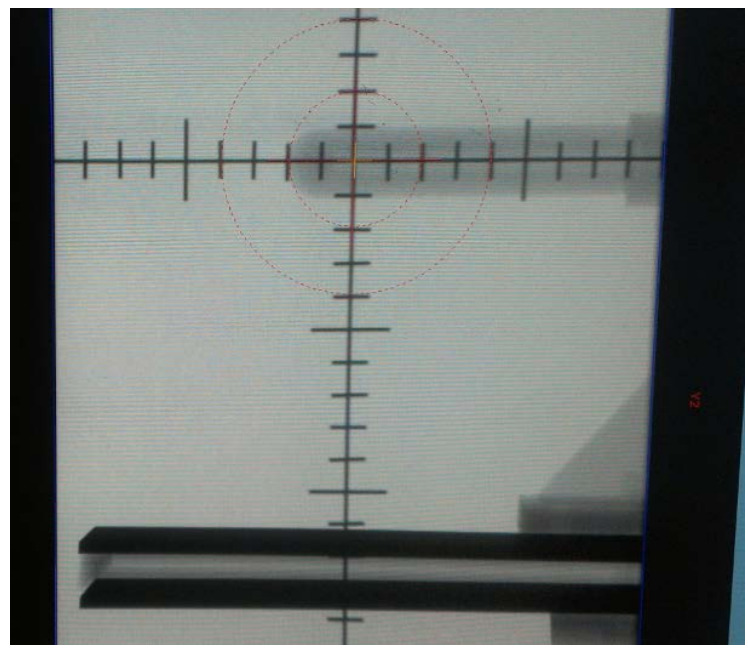

Plate 9. The scales showing distances between the detector holder and the metal plate.

Table 3. Measured dose for a phantom AFTER correction for artifacts on ${ }^{60} \mathrm{Co}$ machine, and a calculated dose of $200 \mathrm{cGy}$.

\begin{tabular}{|c|c|c|c|c|c|c|}
\hline \multicolumn{7}{|c|}{ AFTER the corrections for artifacts $\left({ }^{60} \mathrm{Co}\right.$ beam $)$} \\
\hline \multicolumn{7}{|l|}{ Depth 1} \\
\hline \multirow{2}{*}{ Thickness (mm) } & \multicolumn{3}{|c|}{ Measured dose for a field size (cGy) } & \multicolumn{3}{|c|}{$\begin{array}{l}\% \text { deviation between measured and } \\
\text { calculated }\end{array}$} \\
\hline & $7 \times 7 \mathrm{~cm}^{2}$ & $10 \times 10 \mathrm{~cm}^{2}$ & $15 \times 15 \mathrm{~cm}^{2}$ & $7 \times 7 \mathrm{~cm}^{2}$ & $10 \times 10 \mathrm{~cm}^{2}$ & $15 \times 15 \mathrm{~cm}^{2}$ \\
\hline 0 & 198.820 & 200.000 & 200.620 & -0.59 & 0 & 0.31 \\
\hline 4 & 195.530 & 200.210 & 200.270 & -2.24 & 0.11 & 0.14 \\
\hline 8 & 190.830 & 193.240 & 195.070 & -4.59 & -3.38 & -2.47 \\
\hline 12 & 187.760 & 191.040 & 192.940 & -6.12 & -4.48 & -3.53 \\
\hline 8 (air gap) & 200.500 & 202.740 & 202.850 & 0.25 & 1.37 & 1.42 \\
\hline \multicolumn{7}{|l|}{ Depth 2} \\
\hline \multirow[t]{2}{*}{ Thickness (mm) } & \multicolumn{3}{|c|}{ Measured dose for a field size (cGy) } & \multicolumn{3}{|c|}{$\begin{array}{l}\% \text { deviation between measured and } \\
\text { calculated }\end{array}$} \\
\hline & $7 \times 7 \mathrm{~cm}^{2}$ & $10 \times 10 \mathrm{~cm}^{2}$ & $15 \times 15 \mathrm{~cm}^{2}$ & $7 \times 7 \mathrm{~cm}^{2}$ & $10 \times 10 \mathrm{~cm}^{2}$ & $15 \times 15 \mathrm{~cm}^{2}$ \\
\hline 0 & & & & & & \\
\hline 4 & 199.410 & 199.250 & 201.060 & -0.3 & -0.37 & 0.53 \\
\hline 8 & 189.720 & 191.270 & 193.030 & -5.14 & -4.36 & -3.48 \\
\hline 12 & 186.170 & 189.330 & 192.450 & -6.92 & -5.33 & -3.77 \\
\hline 8 (air gap) & 206.620 & 207.430 & 208.050 & 3.31 & 3.72 & 4.03 \\
\hline
\end{tabular}

at this point varied from $0.25 \%$ to $4.02 \%$ for all depths. Air gap acts as an enhancing medium for gamma rays energy passing through it. This reveals that there are multiple backscatters in the hollow as a result of photoelectric effect, Compton scatter and electron-positron pair production. The beam intensity attenuation increases as the metal thickness increases. The dose decrease at the 
isocentre, $17 \mathrm{~cm}$ deep, is $6.92 \%$ for $12 \mathrm{~mm}$ at shallow depth. Table 3 again shows all the deviations of measured dose to those calculated after the corrections for artifacts by Prowess Panther.

\section{Discussion}

Titanium CT number observed by scanning a tissue characterization phantom is approximately $3070 \mathrm{HU}$. The TPS used extrapolates titanium HU well and gives better simulation for doses around high- $Z$ materials. Prowess could reduce relative dose variation to smaller extend but results could not really match with $\pm 3 \%$ tolerance recommended by ICRU on measured and calculated doses. This is because the gray scale used for a clear viewing of metallic region boundaries to discriminate between water and metal does not give exact boundaries. Also, zooming these boundaries does not give a straight line due to pixels' shapes.

Relative doses measured in the blue water phantom agree with that measured after metal artifacts corrections by the use of a treatment planning system. Increasing a metal thickness means increase in streak artifacts and dose variations at any point along the central axis of the beam. In most cases, percentage decreases for same energy on different field sizes are comparable at each depth. Also regions around metals show dark color, which indicated very low HUs during CT numbers measurements which causes dose distribution inconsistency along the penetrating beam. The boundary between metal and water was hardly recognizable in the CT images and, therefore, artifacts corrections are not really reproducible if metal thickness is unknown. Depth doses were affected by metal presence and thickness of the metal.

\section{References}

[1] Claude, K.P., Tagoe, S.N.A., Schandorf, C. and Amuasi, J.H. (2013) Fabrication of a Tissue Characterization Phantom from Indigenous Materials for Computed Tomography Electron Density Calibration: Peer Reviewed Original Article. The South African Radiographer, 51, 9-17.

[2] Nobah, A., Moftah, B., Tomic, N. and Devic, S. (2011) Influence of Electron Density Spatial Distribution and X-Ray Beam Quality during CT Simulation on Dose Calculation Accuracy. Journal of Applied Clinical Medical Physics, 12, 80-89. https://doi.org/10.1120/jacmp.v12i3.3432

[3] Reft, C., et al. (2003) Dosimetric Considerations for Patients with HIP Prostheses Undergoing Pelvic Irradiation. Report of the AAPM Radiation Therapy Committee Task Group 63. Medical Physics, 30, 1162-1180. https://doi.org/10.1118/1.1565113

[4] Wei, J., Sandison, G.A., Hsi, W.-C., Ringor, M. and Lu, X. (2006) Dosimetric Impact of a CT Metal Artefact Suppression Algorithm for Proton, Electron and Photon Therapies. Physics in Medicine \& Biology, 51, 51-83. https://doi.org/10.1088/0031-9155/51/20/007

[5] Saw, C.B., Loper, A., Komanduri, K., Combine, T., Huq, S. and Scicutella, C. (2005) Determination of CT-to-Density Conversion Relationship for Image-Based Treatment Planning Systems. Medical Dosimetry, 30, 145-148.

https://doi.org/10.1016/j.meddos.2005.05.001 
[6] Shimozato, T., et al. (2010) Dose Distribution near Thin Titanium Plate for Skull Fixation Irradiated by a 4-MV Photon Beam. Journal of Medical Physics, 35, 81-87.

[7] Chatzigiannis, C., et al. (2011) Dose Perturbation in the Radiotherapy of Breast Cancer Patients Implanted with the Magna-Site: A Monte Carlo Study. Journal of Applied Clinical Medical Physics, 12, 58-70. https://doi.org/10.1120/jacmp.v12i2.3295

[8] Miften, M., Wiesmeyer, M., Monthofer, S. and Krippner, K. (2000) Implementation of FFT Convolution and Multigrid Superposition Models in the FOCUS RTP System. Physics in Medicine \& Biology, 45, 817. https://doi.org/10.1088/0031-9155/45/4/301

[9] Bazalova, M., Beaulieu, L., Palefsky, S. and Verhaegen, F. (2007) Correction of CT Artifacts and Its Influence on Monte Carlo Dose Calculations. Medical Physics, 34, 2119. https://doi.org/10.1118/1.2736777 